\title{
Meshed Patch Antenna for Portable UHF Band Radio Communication Devices
}

\author{
Olegs Artamonovs ${ }^{1}$, Guntars Balodis ${ }^{1}$ \\ ${ }^{1}$ Institute of Electronics, Riga Technical University, \\ Azenes St. 12-322, LV-1048 Riga, Latvia \\ olegs.artamonovs@rtu.lv
}

\begin{abstract}
This paper presents a meshed microstrip patch antenna for UHF band radio communications. Meshed elements allow making antenna flexible, light and more suitable for wearable devices. Several antennas, intended for PMR446 band with different meshing configurations, have been designed. Un-meshed, semi-meshed and fully meshed microstrip patch antennas have been compared. The analysis was focused on the best tradeoffs between concentration of conductor, gain and front to back ratio. Results below are showing that more than $50 \%$ of conductor can be removed without significant gain reduction. In some cases the meshing gives a reduction of back radiation. The meshed antenna has smaller dimensions in comparison with the unmeshed antenna on the same frequency.
\end{abstract}

Index Terms-Patch antennas, UHF antennas, UHF communication, mobile antennas, meshed antennas.

\section{INTRODUCTION}

Ultra High Frequency (UHF) band has a high popularity at present. This band is widely used for professional and private communications because of good long distance propagation characteristics. Portable UHF transceivers are equipped with whip antennas. The radiating power of the transmitter can reach $5 \mathrm{~W}$. Portable transceivers, in most cases, when they are used they are located next to the human head and as a result, a lot of RF energy is absorbed in to the tissue of the head. For safe and effective operation, it is recommended to provide at least $100 \mathrm{~mm}$ distance between head and antenna [1]. This recommendation is not always feasible especially for operative and rescue workers. The safety of operators can be improved by using an external wearable antenna. The vest-mounted antennas for UHF military band are presented in [2]-[3]. These antennas are unidirectional and as a result a wearer suffers from RF radiation of the transceiver. A lot of wearable antennas are produced for ISM bands [4]-[6]. These antennas show a high performance and acceptable Specific Absorption Rate (SAR). The short wavelength of ISM $(2.4 \mathrm{GHz})$ band allows to design small antennas with high efficiency. The microstrip patch antenna is the most commonly used type of wearable antennas. This antenna has a high attenuation of the back radiation and high gain in front radiation, as a result high front to back ratio (FB ratio) can be achieved in this antenna. The microstrip patch antennas are not widely used for UHF band (400 MHz-470 MHz) because of the

Manuscript received January 15, 2014; accepted May 8, 2015. large dimensions. Flexible conductive materials can be used for the elements of the microstrip patch antenna. For example, copper gauze is used for UHF wearable antenna [7]. One edge of the patch is grounded, for twice reduction of the length of the antenna. External impedance matching circuits are used for tunning of the antenna. The authors [7] compared a SAR level of dipole and wearable patch antenna. The SAR level of dipole is $4.63 \mathrm{~W} / \mathrm{kg}$, but SAR level of the proposed antenna is $0.05 \mathrm{~W} / \mathrm{kg}$. These results show that wearable microstrip patch antenna has a high onbody performance. The copper gauze which is used for the antenna contains 23 meshes per square centimeter. The authors of this paper assume that the gauze can provide high conductivity, similar to copper plate. The meshed structure is not taken into consideration.

In some cases conductive paints can be used as elements of the antenna. These materials have a better adhesive ability if the coating is meshed. Conductive paints have the ability to shrink after drying up, which is undesirable, because it can cause deformation of the substrate. Meshed structures help to minimize this effect. In addition, meshed structures are more economical and optically transparent in comparison with un-meshed structures. Properties of meshed radiators and ground planes have been studied in [8]. Meshed antennas show a lower gain and lower resonance frequency in comparison with solid antennas with the same dimensions. However, other research [9] shows that meshed structures do not effect significantly on the performances of the antenna. There are two methods of mesh design. In the first method, high density meshes with narrow conductors between cells are used. In the second method, lower density mesh, but with wider conductors are used. In both cases concentration of conductors are the same, but influence on the antenna parameters is different. At present, it is not clear how much of minimum concentration of conductor is acceptable for good performance of the antenna. In order to answer this question several meshed antennas for unlicensed PMR446 band have been designed. This band has 8 channels with $12.5 \mathrm{kHz}$ spacing.

\section{ANTENNA DESIGN}

Unmeshed, semi-meshed and fully-meshed models of microstrip patch antennas were designed for this research. All models of the antennas have an air dielectric between radiator and grounding. Air dielectric was chosen for 
substrate of model to reducing a time of computing. Practically, foam can be used as substrate. Dielectric permitivity of the foam is $1.05-1.3$ which is very close to air. Aluminium was chosen as a conductor. All models of antennas have a coaxial probe feeding with 50 input impedance. The antennas have several geometrical parameters: patch length $-L$, patch width $-W$, feeding offset from edge $-x$, height of antenna $-h$ (distance between radiator and grounding), thickness of conductor $-t$, length of ground plane $-L_{g n d}$, and width of ground plane $W_{\text {gnd }}$. Each geometrical variable has a major influence on the performance of antenna. $L$ - major influence on resonance frequency, $W$ - gain and impedance, $x$ - input resistance, $h-$ bandwidth and gain. Corelation of these parameters on antenna performance is described in more details in [10]. A picture of antenna is given on Fig. 1. Black part is a grounding, white part is a patch radiator.

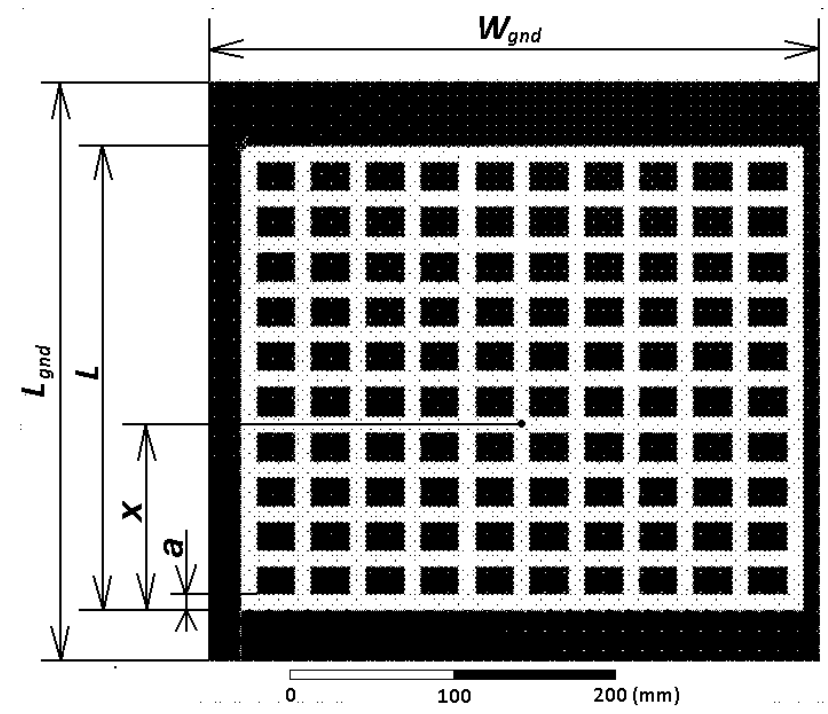

Fig. 1. Dimensions of the semi-meshed antenna.

The semi-meshed antennas have a meshed radiator and unmeshed grounding. The conductor line width is defined by variable $a$. Conductor line width has been deviated from central value during the modelling. Two models of semi meshed antennas were examined in this article. Both models have different central values on the conductor line width. The central value of the first antenna was $a=10 \mathrm{~mm}$ and second model was $a=1 \mathrm{~mm}$.

The full-meshed antennas have meshed radiator and meshed grounding. These antennas have two models with different central values of conductor line width. In the first variant $a=10 \mathrm{~mm}$, in the second variant $a=1 \mathrm{~mm}$. Each radiator and grounding has 100 perforations. Variable $q$ defines quantity of the perforations. In all meshed antennas $q=100$. The relative area of conductor in meshed antennas can be expressed by concentration $N$. The concentration of the conductor is a relationship between the area of conductor and the patch area (1)

$$
N=\frac{a(\sqrt{q}+1)(L+W-a(\sqrt{q}-1))}{L \times W} \times 100 .
$$

It can be noted that concentration $N$ depends on conductor line width and dimensions of patch ( $W$ and $L$ ). During a parametric modeling, the variable $a$ was being changed.

\section{ANTENNA MODELLING}

\section{A. Unmeshed Antenna}

Unmeshed rectangular patch antenna was created in order to define the maximum values of the main parameters of the antenna. This antenna will be referenced for further analysis. Dimensions of the antenna of this experiment are as per the following: $L=308.8 \mathrm{~mm}, W=340 \mathrm{~mm}, x=108 \mathrm{~mm}$. The grounding dimensions are as per the following: $W_{\text {gnd }}=$ $360 \mathrm{~mm}, L_{\text {gnd }}=360 \mathrm{~mm}$. Height and thickness are constant for all models, $h=10 \mathrm{~mm}$ and $t=0.1 \mathrm{~mm}$ respectively. During the modelling the most important characteristics of the antenna have been obtained. These characteristics are as per the following: return losses (S11), gain, FB ratio. Ansoft HFSS software was used for modeling. The antenna was optimised in order to get a minimal return losses at $446 \mathrm{MHz}$ frequency. Return losses should be less than $10 \mathrm{~dB}$, otherwise a lot of power would be reflected back to transmitter. After simulation $-25 \mathrm{~dB}$ return losses, $9.2 \mathrm{~dB}$ gain, $10.3 \mathrm{~dB} \mathrm{FB}$ ratio and $6 \mathrm{MHz}$ bandwidth has been resulted.

\section{B. Semi-Meshed Antenna}

Input parameters of the first model of the semi-meshed antenna are as per the following: $a=10 \mathrm{~mm}$ and concentration of conductor $(N=60 \%)$ The dimensions of the semi-meshed antenna are the same as unmeshed antenna with one exception: patch lenght has a new value $L=$ $280 \mathrm{~mm}$. A central value of line width is $a=10 \mathrm{~mm}$. The parametric analysis with different values of the conductor line width has been done. Line width had been changing through the range from $2 \mathrm{~mm}$ to $18 \mathrm{~mm}$, with $4 \mathrm{~mm}$ step. Return losses (S11) of the semi-meshed antennas are shown on Fig. 2. It is apparent, that line width has high influence on resonance frequency. The resonance frequency reduces as $a$ decreases. As a conductor line width is changed in the range between $6 \mathrm{~mm}-18 \mathrm{~mm}$, s11 stays on the acceptable level $(<-10 \mathrm{~dB})$. However, it can be seen in Fig. 2, when $a=2 \mathrm{~mm}$, the return losses became too high $(-10 \mathrm{~dB})$. It is not acceptable, because the antenna is unmatched and as a result is not effective.

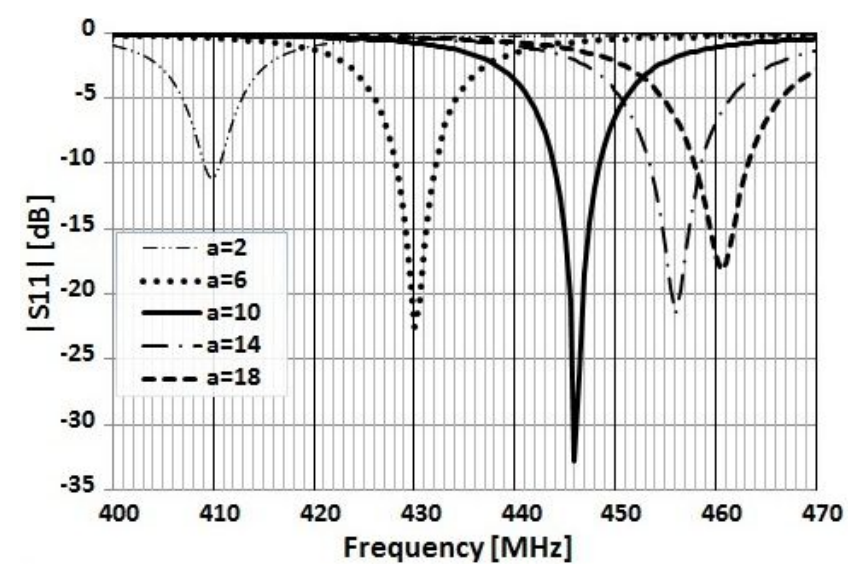

Fig. 2. Return losses of the semi-meshed antenna $(a=10 \mathrm{~mm})$.

The essential parameters of the antenna are shown on Fig. 3. The bottom $\mathrm{X}$-axis represents the variation of the conductor line width during the modeling. The 
concentration of conductor is shown on the top X-axis. The values of concentration on the graph are calculated by using (1). The concentration varies from $14 \%$ to $99 \%$. The left $\mathrm{Y}$-axis shows the gain of antenna, but the right $\mathrm{Y}$-axis shows the FB ratio. The central $\mathrm{Y}$-axis shows the relative shift of resonance frequency. Zero of frequency shift corresponds to $446.0 \mathrm{MHz}$ frequency.

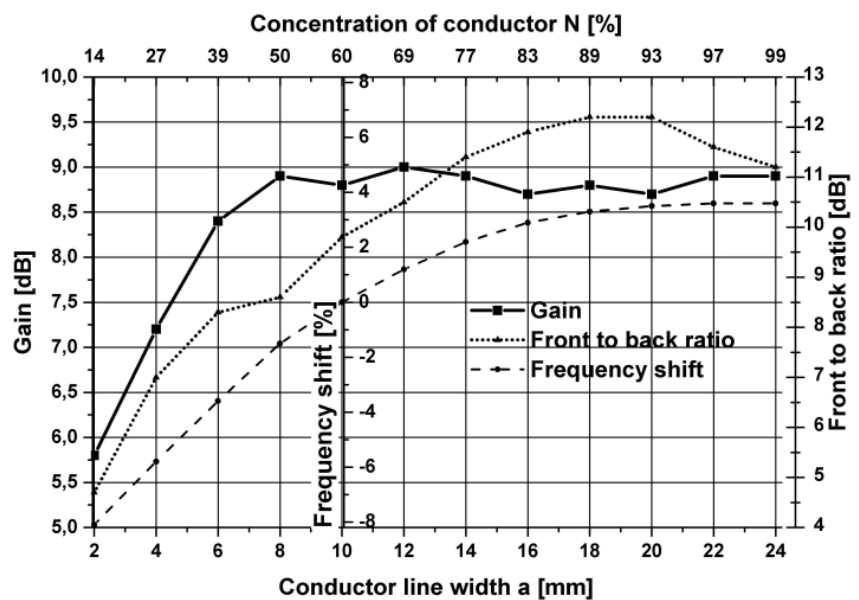

Fig. 3. Results of simulation of the semi-meshed ( $a=10 \mathrm{~mm})$.

From these graphs we can notice that gain of the antenna suffers seriously if concentration is lower than $40 \%$. However, the antenna has a stable value of gain when the concentration of conductor is higher than $50 \%$. The maximal value of gain is $9.0 \mathrm{~dB}$ which is very close to gain of unmeshed antenna $(9.2 \mathrm{~dB})$. Maximum of FB ratio of the semi meshed antenna is $12 \mathrm{~dB}$ which is better in comparison with referenced unmeshed antenna which has $10.3 \mathrm{~dB}$. The resonance frequency is practically constant when the concentration $N$ is within $80 \%-99 \%$. It is noticeable that patch radiator can lose up to $20 \%$ of the area of the conductor without any negative impact on the characteristics of the antenna. However, it is very important to keep the conductor uniform.

The performance of the semi-meshed antenna has significant deterioration when the conductor line width is close to $2 \mathrm{~mm}$ instead of the optimal $10 \mathrm{~mm}$. When $a=$ $2 \mathrm{~mm}$ the antenna has a strong detuning. We suggest that antenna should be re-optimized for further analysis. For this purpose we made the second model of semi-meshed antenna with a new reference value of conductor line width. In the new model $a=1 \mathrm{~mm}$ instead of $10 \mathrm{~mm}$.

The dimensions of second model of the semi-meshed antenna were reduced to get resonance on $446.0 \mathrm{MHz}$ frequency. The essential influence on resonance frequency gives length on the patch. The length was reduced by $24 \mathrm{~mm}$. Also, other geometric parameters were reduced to save balance between dimensions. The dimensions are following: $L=256 \mathrm{~mm}, W=280 \mathrm{~mm}$ and $x=108 \mathrm{~mm}$, $L_{\text {gnd }}=320 \mathrm{~mm}, W_{\text {gnd }}=320 \mathrm{~mm}$. Conductor line width have been changed through the range from $0.2 \mathrm{~mm}$ to $2 \mathrm{~mm}$, with a step $0.4 \mathrm{~mm}$. The resonance characteristics are given on Fig. 4. It is noticeable that values of return losses (S11) are admissible for all the models. It means that input impedance of the antenna is practically independent from the conductor line width. By changing width of the conductive line 10 times, the antenna stays well matched. Return losses S11 =
$-15 \mathrm{~dB}$, when width of the line is minimal $(a=0.2 \mathrm{~mm})$, this corresponds only to $3 \%$ of the power loss. The characteristics of the semi-meshed antenna with more narrow line width $(a=1 \mathrm{~mm})$ are more stable in comparison with antenna which has a wide line width $(a=10 \mathrm{~mm})$.

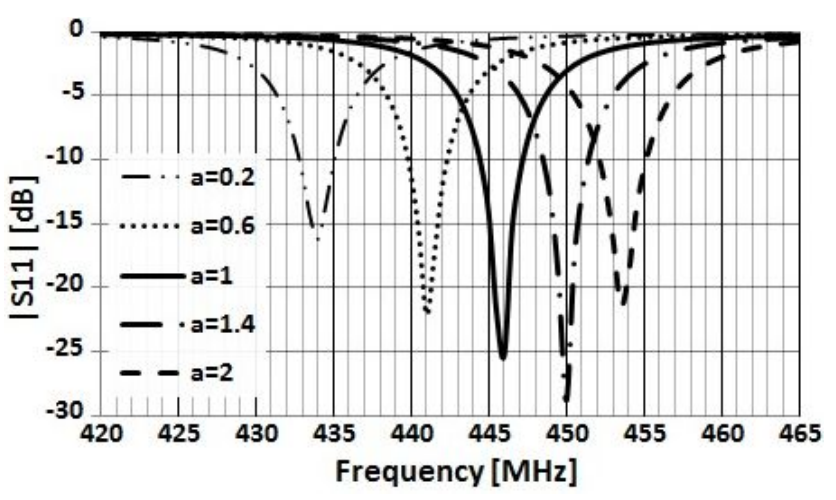

Fig. 4. Return losses of the semi-meshed antenna $(a=1.0 \mathrm{~mm})$.

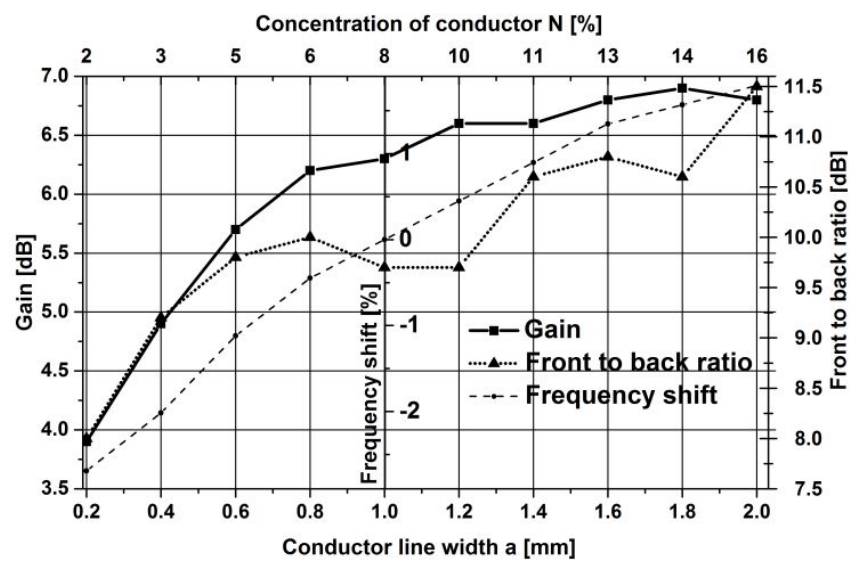

Fig. 5. Results of simulation of the semi-meshed antenna $(a=1.0 \mathrm{~mm})$.

The most significant parameters of the second model of the semi-meshed antenna are given on Fig. 5. The concentration of the conductor varies from $2 \%$ to $16 \%$. We observe that gain and $\mathrm{FB}$ ratio are growing with concentration of the conductor. At the maximum concentration of the conductor the maximal gain and FB ratio can be obtained. This observation tells us, that reduction of concentration more than on $16 \%$ has a negative effect on the performance of the antenna. When the line width is minimal a $=0.2 \mathrm{~mm}$, then a gain is only $4 \mathrm{~dB}$ and FB-ratio is $8 \mathrm{~dB}$ which is not enough for microstrip antenna and it can't be used for practical design.

\section{Full-meshed Antenna}

The experiments on the semi-meshed antenna show that the characteristics of antenna are acceptable if $a>1.0 \mathrm{~mm}$. Now let's consider full-meshed antenna. This antenna consists of meshed patch and meshed ground plane. We have run the similar experiment as we have run with semimeshed antenna. The first model is designed with width of conductor line $a=10 \mathrm{~mm}$ for patch and ground. The dimensions of the antenna are as per the following: $L=$ $262.5 \mathrm{~mm}, W=300 \mathrm{~mm}, x=90 \mathrm{~mm}, L_{g n d}=320 \mathrm{~mm}, W_{\text {gnd }}=$ $320 \mathrm{~mm}$. We have performed a parametric modeling of antenna where the conductor line width is variable. The resonance characteristic of the full-meshed antenna is very similar to Fig. 2 and Fig. 4 So we did not show it again. 
Let's concentrate on Fig. 6.The graph shows that the FB ratio has a peak at $20 \mathrm{~dB}$ and antenna has high gain (> $8.0 \mathrm{~dB}$ ) if the line width is $14 \mathrm{~mm}-16 \mathrm{~mm}$ and $80 \%-86 \%$ concentration respectively. We have got a combination of high gain and high FB ratio as a result the influence on the human body is minimized. The full-meshed antenna is more sensitive to detuning in comparison with semi-meshed antenna. As the conductor line width was changed through $2 \mathrm{~mm}-24 \mathrm{~mm}$ range, the central frequency of the patch changed by nearly $18 \%$. The best parameters of the full meshed antenna was acheived when $a=15 \mathrm{~mm}$.

Figure 7 shows the results of the modelling of the fullmeshed antenna, which has narrow $(0.2 \mathrm{~mm}-2 \mathrm{~mm})$ conductor. From the graph we can see that both parameters gain and FB ratio are decreasing as conductor line width and concentration are decreasing as well. The antenna became ineffective if $a<2 \mathrm{~mm}$.

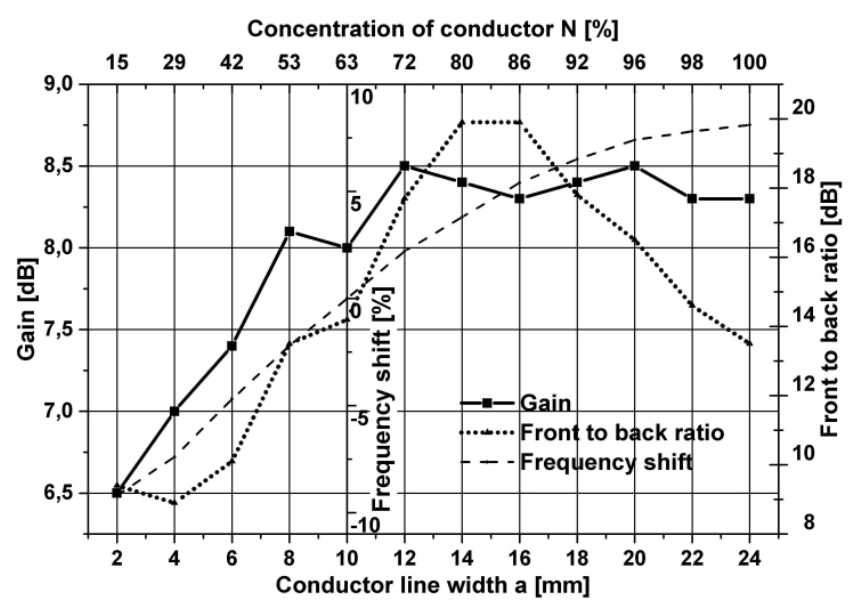

Fig. 6. Results of simulation of the full-meshed antenna $(a=10 \mathrm{~mm})$.

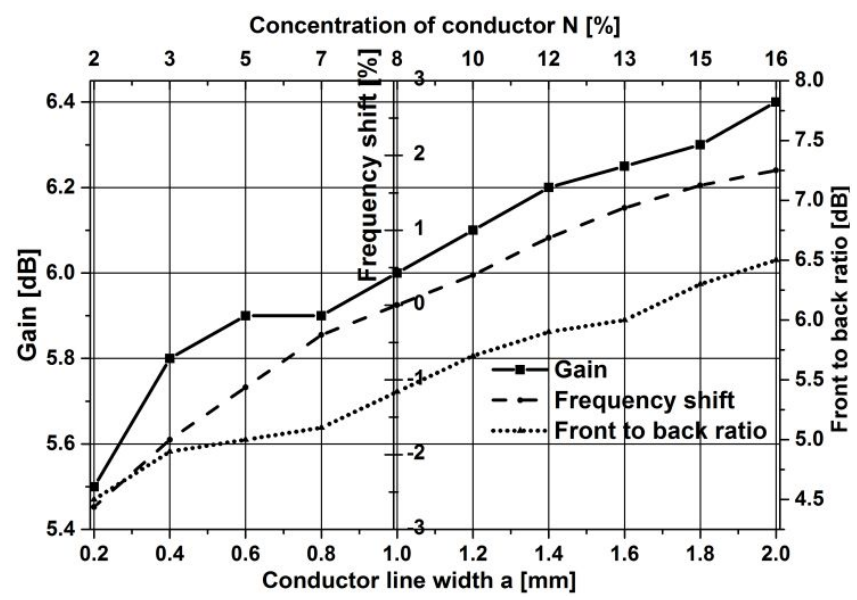

Fig. 7. Results of simulation the full-meshed antenna ( $a=1 \mathrm{~mm})$.

\section{CONCLUSIONS}

The results of this work show that it is possible to make a meshed microstrip patch antenna for UHF band radio communications. The best result was achieved with full meshed antenna when the conductor line width was $15 \mathrm{~mm}$. In this case the concentration of conductor is about $80 \%$. The full-meshed structure has positive effect to FB ratio of the antenna. The FB ratio is $10 \mathrm{~dB}$ higher in comparison with solid rectangular antenna. The gain is about $8.5 \mathrm{dBi}$, which is much better than dipole antenna. The high attenuation of back radiation can provide a low SAR level. The meshed antennas give an opportunity to economise materials and demonstrate better flexibility. The meshed structure of patch antenna allows to make the antennas more compact without using any special substrate with high dielectric permittivity. Additional meshing allows to minimize a negative effect of shrinkage of conductive paints after drying up.

\section{REFERENCES}

[1] N. Kogo, T. Ikeda, "Antenna performance of push-to-talk transceiver in VHF and UHF tands considering impedance matching", in Proc. IEEE Int. Symp. on Electromagnetic Theory, 2013, pp. 358-361.

[2] G. Y. Lee, D. Psychoudakis, C. C. Chen, J. L. Volakis, "Omnidirectional vest-mounted body-worn antenna system for UHF operation", IEEE Antennas Wireless Propagat. Lett., vol. 10, pp. 581583, 2011. [Online]. Available: http://dx.doi.org/10.1109/LAWP. 2011.2158381

[3] D. Psychoudakis, G. Y. Lee, C. C. Chen, J. L. Volakis, "Military UHF body-worn antennas for armored vests", in Proc. Eu. Conf. Antenna Propagat, 2010, pp. 1-4.

[4] T. Kennedy, P. Fink, A. Chu, N. Champagne, G. Lin, "Body-worn etextile antennas: the good, the low-mass, and the conformal", IEEE Trans. Antennas Propagat., vol. 57, no. 4, pp. 910-918, 2009. [Online]. Available: http://dx.doi.org/10.1109/TAP.2009.2014602

[5] R. Moro, S. Agneessens, H. Rogier, M. Bozzi, "Wearable textile antenna in substrate integrated waveguide technology", IEEE Electron. Lett. (II), vol. 48, no. 16, pp. 985-987, 2012. [Online]. Available: http://dx.doi.org/10.1049/el.2012.2349

[6] S. J. Chen, T. Kaufmann, C. Fumeaux, "Wearable textile microstrip patch antenna for multiple ISM band communications", in Proc. Symp. IEEE Antennas. Propagat. Society, 2013, pp. 1860-1861. [Online]. Available: http://dx.doi.org/10.1109/aps.2013.6711588

[7] H. Wang, Z. Zhang, Y. Li, Z. Feng. "A dual-resonant shorted patch antenna for wearable application in $430 \mathrm{MHz}$ Band", IEEE Trans. Antennas Propagat., vol. 61, no. 12, pp. 6195-6200, 2013. [Online]. Available: http://dx.doi.org/10.1109/TAP.2013.2282044

[8] G. Clasen, R. Langley, "Meshed patch antennas", IEEE Trans. Antennas and Propagat., vol. 52, no. 6, pp. 1412-1416, 2004. [Online]. Available: http://dx.doi.org/10.1109/TAP.2004.830251

[9] M. Shuang, G. H. Yang, E. C. Wang, C. F. Zhou, "Microstrip antenna design based on grid structure for optical transmission", in Proc. IEEE 5th Global Symp. on millimeter waves, 2012, pp. 127-130. [Online]. Available: http://dx.doi.org/10.1109/gsmm.2012.6313987

[10] Microstrip antenna design handbook, Artech House, Inc., 2001, pp. 265-289. 